\title{
A 4D Statistical Shape Model for Automated Segmentation of Lungs with Large Tumors
}

\author{
Matthias Wilms, Jan Ehrhardt, and Heinz Handels \\ Institute of Medical Informatics, University of Lübeck, Lübeck, Germany \\ wilms@imi.uni-luebeck.de
}

\begin{abstract}
Segmentation of lungs with large tumors is a challenging and time-consuming task, especially for 4D CT data sets used in radiation therapy. Existing lung segmentation methods are ineffective in these cases, because they are either not able to deal with large tumors and/or process every $3 \mathrm{D}$ image independently neglecting temporal information. In this paper, we present a approach for model-based 4D segmentation of lungs with large tumors in 4D CT data sets. In our approach, a 4D statistical shape model that accounts for inter- and intra-patient variability is fitted to the $4 \mathrm{D}$ image sequence, and the segmentation result is refined by a $4 \mathrm{D}$ graph-based optimal surface finding. The approach is evaluated using 10 4D CT data sets of lung tumor patients. The segmentation results are compared with a standard intensity-based approach and a $3 \mathrm{D}$ version of the presented model-based segmentation method. The intensity-based approach shows a better performance for normal lungs, however, fails in presence of large lung tumors. Although overall performance of $3 \mathrm{D}$ and $4 \mathrm{D}$ model-based segmentation is similar, the results indicate improved temporal coherence and improved robustness with respect to the segmentation parameters for the $4 \mathrm{D}$ model-based segmentation.
\end{abstract}

\section{Introduction}

Breathing-induced tumor motion represents a major challenge in radiation therapy of lung cancer. Patient-specific information about the respiratory dynamics, estimated by using spatio-temporal 4D CT data sets and non-linear image registration techniques, can therefore help to optimize the treatment planning and the delivery process [1. To confine motion estimation and subsequent analysis steps (e.g., tumor detection and tracking) to the lungs, segmentations of the lung tissue in all 3D images of a $4 \mathrm{D}$ sequence are needed.

Many approaches dealing with the automatic segmentation of healthy lungs in 3D CT images have been proposed (e.g., [2] ]. These methods mainly take advantage of the large density difference between the air-filled lungs and surrounding tissue and therefore frequently fail to include areas of high density abnormalities (e.g., tumors) into the segmentation. As a consequence, several groups have suggested algorithms specifically designed to handle CT images of pathological lungs by incorporating prior knowledge to guide the segmentation process. For example, Sluimer et al. [4] and van Rikxoort et al. [5] employed 
atlas-based techniques for the segmentation of lungs with arbitrary pathologic abnormalities. They were able to significantly increase segmentation accuracy, but the required non-linear atlas-to-image registration process was very time consuming. Sun et al. presented a time-efficient method especially aimed to segment lungs with large tumors using a robust active shape model [6]. Their results are very promising, but their approach, like other approaches, can only handle single $3 \mathrm{D}$ images. In the case of $4 \mathrm{D}$ data sets, methods processing all individual 3D images separately ignore the temporal information included in spatio-temporal 4D data: We hypothesize that including temporal information improves robustness of the segmentation process and temporal consistency of the results.

The aim of this work is the temporally consistent segmentation of lungs with large tumors in 4D CT data sets. Our method is based on the work of Perperidis et al. [7, who used a 4D statistical shape model (4D-SSM) for 4D cardiac image segmentation. This 4D-SSM accounts for both changes of the organ shape caused by inter-patient variability and shape changes due to cardiac (or in our case, respiratory) dynamics (intra-patient variability). We present a novel 4D fitting algorithm for this 4D-SSM and refine the segmentation results by using 4D graph-based optimal surface finding. In an evaluation, the results of our 4D approach are compared to segmentations obtained by using standard 3D-SSMs, as well as results of a standard intensity-based lung segmentation algorithm.

\section{Method}

First, we generate a 4D-SSM for each lung based on $N_{p}$ segmented 4D CT image sequences of different patients (Section 2.1). Each image sequence is assumed to consist of $N_{j}$ 3D image volumes $I_{p, j}: \Omega \rightarrow \mathbb{R}\left(\Omega \subset \mathbb{R}^{3}\right)$, reconstructed at corresponding phases $j$ of the breathing cycle, e.g. end-expiration (EE), midinspiration (MI), end-inspiration (EI), and mid-expiration (ME). For segmentation, the generated model is simultaneously adapted to all 3D images of an unseen 4D CT data set (Section 2.2). Afterwards, all segmentations are refined using a graph-based post-processing method (Section 2.3).

\subsection{Building a 4D Statistical Shape Model}

The first step in building a statistical shape model (SSM) based on a training set of $N=N_{p} N_{j}$ complete lung shapes obtained from segmented 4D CT image sequences is to establish correspondence between all shapes. This is achieved by propagating $M$ pseudo-landmarks from an automatically landmarked atlas to all other shapes of the training set. After the generation and landmarking (by means of a surface triangulation and curvature-based mesh simplification) of an average lung shape atlas, landmark propagation is done by using non-linear transformations obtained from atlas-patient and intra-patient registrations of the images performed with a non-linear diffeomorphic registration method [8].

Let $\left\{\boldsymbol{q}_{p, j} \in \mathbb{R}^{3 M} \mid p=1, \ldots, N_{p} ; j=1, \ldots, N_{j}\right\}$ denote the set of $N$ aligned training shapes. Each shape vector $\boldsymbol{q}_{p, j}=\left[\boldsymbol{p}_{p, j, 1}^{T}, \ldots, \boldsymbol{p}_{p, j, M}^{T}\right]^{T}$ consists of a 
concatenation of $M$ landmarks $\boldsymbol{p}_{p, j, k} \in \Omega$. Principal component analysis (PCA) performed on this shape vectors yields a common 3D-SSM

$$
S_{3 D}(\boldsymbol{b}, \varphi)=\varphi(\overline{\boldsymbol{q}}+\boldsymbol{P b}), \text { with } \quad \overline{\boldsymbol{q}}=\frac{1}{N} \sum_{p=1}^{N_{p}} \sum_{j=1}^{N_{j}} \boldsymbol{q}_{p, j},
$$

where $\boldsymbol{P}$ denotes a matrix whose columns are orthonormal eigenvectors $\boldsymbol{e}_{i}$ of covariance matrix $\boldsymbol{C}=1 / N \sum_{p=1}^{N_{p}} \sum_{j=1}^{N_{j}}\left(\boldsymbol{q}_{p, j}-\overline{\boldsymbol{q}}\right)\left(\boldsymbol{q}_{p, j}-\overline{\boldsymbol{q}}\right)^{T}$ with eigenvalues $\lambda_{i}<\lambda_{i+1}$ [9]. The model is parameterized by shape parameter vector $\boldsymbol{b}$ and similarity transformation $\varphi$. As a consequence of using all shapes of all patients to estimate $\boldsymbol{C}$, the resulting eigenmodes explain both inter- and intra-patient variability. This leads to $N_{j}$ different and independent sets of model parameters $\{\boldsymbol{b}, \varphi\}$ to describe a lung's shape during a breathing cycle, making the model unsuitable for $4 \mathrm{D}$ segmentation. We therefore propose the application of a socalled 4D-SSM based on the work of Perperidis et al. [7] and defined by

$$
S_{4 D}\left(\boldsymbol{b}_{\text {inter }}, \boldsymbol{b}_{\text {intra }}^{j}, \varphi\right)=\varphi\left(\overline{\boldsymbol{q}}+\boldsymbol{P}_{\text {inter }} \boldsymbol{b}_{\text {inter }}+\boldsymbol{P}_{\text {intra }} \boldsymbol{b}_{\text {intra }}^{j}\right),
$$

which describes a patient's lung shape as a combination of a fixed patient-specific part (given by $\boldsymbol{b}_{\text {inter }}$ and $\varphi$ ) and a varying part depending on the breathing phase $j$ (weighted by $\boldsymbol{b}_{\text {intra }}^{j}$ ). $\boldsymbol{P}_{\text {inter }}$ and $\boldsymbol{P}_{\text {intra }}$ denote matrices of orthonormal eigenvectors defining subspaces of $\mathbb{R}^{3 M}$ accounting for inter- and intra-patient variability, respectively. Separate PCAs performed on the covariance matrices $\boldsymbol{C}_{\text {inter }}$ and $\boldsymbol{C}_{\text {intra }}$ yield the eigenvectors defining both subspaces. $\boldsymbol{C}_{\text {inter }}$ explains the variability across the different patients and is given by

$$
\boldsymbol{C}_{\text {inter }}=\frac{1}{N_{p}} \sum_{p=1}^{N_{p}}\left(\overline{\boldsymbol{q}}_{p}-\overline{\boldsymbol{q}}\right)\left(\overline{\boldsymbol{q}}_{p}-\overline{\boldsymbol{q}}\right)^{T}, \text { with } \quad \overline{\boldsymbol{q}}_{p}=\frac{1}{N_{j}} \sum_{j=1}^{N_{j}} \boldsymbol{q}_{p, j} .
$$

Accordingly, $\boldsymbol{C}_{\text {intra }}$ describes the shape differences across the respiratory cycle as deviations from the mean shape of each patient $p$ :

$$
\boldsymbol{C}_{\text {intra }}=\frac{1}{N_{p} N_{j}} \sum_{p=1}^{N_{p}} \sum_{j=1}^{N_{j}}\left(\boldsymbol{q}_{p, j}-\overline{\boldsymbol{q}}_{p}\right)\left(\boldsymbol{q}_{p, j}-\overline{\boldsymbol{q}}_{p}\right)^{T} .
$$

\subsection{Fitting the Model to an Image Sequence}

The simultaneous lung segmentation in all $N_{j}$ 3D images $I_{j}, j \in\left\{1 \ldots, N_{j}\right\}$, of a 4D CT data set using a 4D-SSM (eq. 2) consists of finding parameters $\left\{\tilde{\boldsymbol{b}}_{\text {inter }}, \tilde{\boldsymbol{b}}_{\text {intra }}^{1}, \ldots, \tilde{\boldsymbol{b}}_{\text {intra }}^{N_{j}}, \tilde{\varphi}\right\}$, such that $\tilde{\boldsymbol{r}}_{j}=S_{4 D}\left(\tilde{\boldsymbol{b}}_{\text {inter }}, \tilde{\boldsymbol{b}}_{\text {intra }}^{j}, \varphi\right)$ is a good approximation of lung shape $\boldsymbol{r}_{j} \in \mathbb{R}^{3 M}$ implicitly encoded in $I_{j}$. When using the common sum of squared distances between corresponding model landmarks and image points as a measure, this can be formulated as the optimization problem

$$
\sum_{j=1}^{N_{j}}\left\|\boldsymbol{r}_{j}-S_{4 D}\left(\boldsymbol{b}_{\text {inter }}, \boldsymbol{b}_{\text {intra }}^{j}, \varphi\right)\right\|^{2} \longrightarrow \min
$$


which we divide into

$$
\begin{aligned}
\left(\tilde{\boldsymbol{b}}_{\text {inter }}, \tilde{\varphi}\right) & =\underset{\boldsymbol{b}_{\text {inter }, \varphi}}{\operatorname{argmin}}\left\|\frac{1}{N_{j}} \sum_{j=1}^{N_{j}} \boldsymbol{r}_{j}-S_{4 D}\left(\boldsymbol{b}_{\text {inter }}, 0, \varphi\right)\right\|^{2} \text { and } \\
\left(\tilde{\boldsymbol{b}}_{\text {intra }}^{j}\right) & =\underset{\boldsymbol{b}_{\text {intra }}^{j}}{\operatorname{argmin}}\left\|\boldsymbol{r}_{j}-S_{4 D}\left(\tilde{\boldsymbol{b}}_{\text {inter }}, \boldsymbol{b}_{\text {intra }}^{j}, \tilde{\varphi}\right)\right\|^{2} .
\end{aligned}
$$

Eq. (6) is motivated by the assumption that patient-specific shape properties independent of the breathing motion can be described by the patient's mean shape (eq. (3) ). In contrast to [7], both problems are minimized by an alternating iterative optimization scheme based on the active shape model (ASM) algorithm 9] without a heuristic pre-initialization of the intra-patient parameters $\boldsymbol{b}_{\text {intra }}^{j}$ :

1. Initial placing of mean shape $S_{4 D}(0,0, \varphi)$. The initial $\varphi$ is determined by a heuristic based on the detected bronchial tree [10.

2. For all $j \in\left\{1 \ldots, N_{j}\right\}$ : Displace each landmark $\boldsymbol{p}_{j, k}$ of model instance $S_{4 D}\left(\tilde{\boldsymbol{b}}_{\text {inter }}, \tilde{\boldsymbol{b}}_{\text {intra }}^{j}, \tilde{\varphi}\right)$ to better match the corresponding lung surface in $I_{j}$. The displaced landmarks $\hat{\boldsymbol{p}}_{j, k}$ form a candidate shape vector $\boldsymbol{r}_{j} \in \mathbb{R}^{3 M}$.

3. The mean candidate shape vector $\overline{\boldsymbol{r}}=1 / N_{j} \sum_{j=1}^{N_{j}} \boldsymbol{r}_{\boldsymbol{j}}$ is used to determine $\tilde{\varphi}$ and $\tilde{\boldsymbol{b}}_{\text {inter }}$ (see eq. (6) ) by the minimization of $\|\overline{\boldsymbol{r}}-\varphi(\overline{\boldsymbol{q}})\|^{2}$, and $\tilde{\boldsymbol{b}}_{\text {inter }}=$ $\boldsymbol{P}_{\text {inter }}^{T}\left(\tilde{\varphi}^{-1}(\overline{\boldsymbol{r}})-\overline{\boldsymbol{q}}\right)$.

4. Repeat step 2. Each new $\boldsymbol{r}_{j}$ is used to determine a corresponding breathingrelated $\tilde{\boldsymbol{b}}_{\text {intra }}^{j}$. Solving (7) yields $\tilde{\boldsymbol{b}}_{\text {intra }}^{j}=\boldsymbol{P}_{\text {intra }}^{T}\left(\tilde{\varphi}^{-1}\left(\boldsymbol{r}_{j}\right)-\overline{\boldsymbol{q}}-\boldsymbol{P}_{\text {inter }} \tilde{\boldsymbol{b}}_{\text {inter }}\right)$.

5. Steps 2-4 are repeated until convergence.

A displaced landmark's position $\hat{\boldsymbol{p}}_{j, k}=\boldsymbol{p}_{j, k}+\boldsymbol{n}_{j, k} \delta s_{j, k}$ is determined by choosing the optimal position

$$
s_{j, k}=\underset{l=-L, \ldots,+L}{\operatorname{argmin}} F_{j, k}\left(\boldsymbol{p}_{j, k}+\boldsymbol{n}_{j, k} \delta l\right)
$$

on a sampled 1D intensity profile of $2 L+1$ points at intervals of $\delta$ along the unit surface normal $\boldsymbol{n}_{j, k}$. Each sampling point is evaluated by

$$
F_{j, k}(\boldsymbol{x})= \begin{cases}1 & \text { if } I_{j}(\boldsymbol{x})>-100 \mathrm{HU} \\ 1-\frac{\max \left\{0, \boldsymbol{n}_{j, k}^{T} \nabla I_{j}(\boldsymbol{x})\right\}}{g_{\max , j}} & \text { otherwise }\end{cases}
$$

where $g_{\max , j}$ is the maximum gradient magnitude in $I_{j}$. The value $F_{j, k}(\boldsymbol{x})$ is inversely related to the likelihood that $\boldsymbol{x}$ is a point on the lung's surface.

Success of the model fitting largely depends on the selected displaced landmarks. If many landmarks are detected at positions representing transitions from healthy lung parenchyma to tumor tissue, the outlined least squares approach will fail to recover the true lung shape. We try to avoid this by assuming an initial position close to the lung shape, and therefore use only short profiles. 


\subsection{Optimal Surface Finding}

The final part of our segmentation approach aims at integrating patient-specific shape variations, not described by $4 \mathrm{D}-\mathrm{SSM}$ s built from usually small training sets. Therefore, the $N_{j}$ shapes $\left\{\tilde{\boldsymbol{r}}_{j}\right\}$ resulting from the model fitting step are deformed to better match the image data, while maintaining the established spatial and temporal consistency (optimal surface finding). Let $\tilde{R}_{j}=\left(V_{j}, E_{j}\right)$ denote the triangulated surface mesh of shape vector $\tilde{\boldsymbol{r}}_{j}$ with vertex set $V_{j}$ and edge set $E_{j}$. The deformation of all surfaces is, as in Sec. 2.2. achieved by displacing every vertex $\boldsymbol{p}_{j, k} \in V_{j}$ along its surface normal to a position $\hat{\boldsymbol{p}}_{j, k}=\boldsymbol{p}_{j, k}+\boldsymbol{n}_{j, k} \delta s_{j, k}$. In contrast to the model fitting step, where all displacements $\left\{s_{j, k} \in[-L . .+L]\right\}$ are detected independently, globally optimal solutions are needed to preserve the consistencies mentioned above. The task of determining spatially consistent refined segmentations can be defined as the optimization problem

$$
\begin{aligned}
& \sum_{j=1}^{N_{j}} \sum_{k=1}^{M} F_{j, k}\left(\boldsymbol{p}_{j, k}+\boldsymbol{n}_{j, k} \delta s_{j, k}\right)+\sum_{j=1}^{N_{j}} \sum_{\left[\boldsymbol{p}_{j, k}, \boldsymbol{p}_{j, m}\right] \in E_{j}} a\left|s_{j, k}-s_{j, m}\right| \stackrel{\left\{s_{j, k}\right\}}{\longrightarrow} \min \\
& \text { subject to } \quad \forall j \in\left\{1, \ldots, N_{j}\right\} \forall\left[\boldsymbol{p}_{j, k}, \boldsymbol{p}_{j, m}\right] \in E_{j}:\left|s_{j, k}-s_{j, m}\right| \leq \Delta_{3 D}
\end{aligned}
$$

where $\Delta_{3 D}$ is the parameter of a hard smoothness constraint specifying how many steps adjacent vertices are allowed to shift against each other on their sampled profiles, while constant $a$ penalizes every shift (soft smoothness constraint). Both constraints aim to prevent large deviations from the prior shapes $\left\{\tilde{\boldsymbol{r}}_{j}\right\}$. A globally optimal solution of eq. (10) can be obtained by computing the minimum-closed-set of a directed arc-weighted graph build from all sampled profiles using a max-flow/min-cut algorithm [11]. For our 4D segmentation approach, we also try to maintain the established temporal consistency by incorporating an additional hard smoothness constraint into eq. (10), where $\Delta_{4 D}$ limits the shifting of temporally corresponding vertices:

$$
\forall m \in\left\{1, \ldots, N_{j}\right\} \forall n \in\left\{m, \ldots, N_{j}\right\} \forall k \in\{1, \ldots, M\}:\left|s_{m, k}-s_{n, k}\right| \leq \Delta_{4 D} .
$$

\section{Experiments and Results}

$124 \mathrm{D}$ CT data sets of healthy lungs with $N_{j}=10$ phases are used to build 3DSSMs (eq. (1D) and 4D-SSMs (eq. (2) ) for left and right lung separately $(N=120$ shapes with $M \approx 2000$ landmarks). The most significant inter-patient eigenmode of the 4D-SSM describes lung shapes from high and thin to low and broad, while the most significant intra-patient mode explains most of the breathing-related volume changes.

$104 \mathrm{D}$ CT data sets of lung cancer patients (each containing 7-14 3D images) are used for the evaluation. All images had a size of $512 \times 512 \times 126-467$ voxel with a voxel size between $0.94 \times 0.94 \times 1.5 \mathrm{~mm}^{3}$ and $0.97 \times 0.97 \times 3.0 \mathrm{~mm}^{3}$. Out of the 20 different lungs, 8 were without abnormalities (normal lungs), 7 contained small tumors $<13 \mathrm{~cm}^{3}$, and 5 contained large tumors $>13 \mathrm{~cm}^{3}$ adhering to nonlung structures. Manual segmentations were available for the breathing phases 
Table 1. Performance comparison between 4D-Seg, 3D-Seg, standard intensity-based (Conv), and combined (Comb) segmentation approaches. Results averaged over all processed lungs of each group (normal \& small tumors, large tumors), given as $\mu \pm \sigma$. Error metrics: Jaccard coefficient $J(A, B)$, symmetric mean surface distance $\bar{D}(A, B)$, and the symmetric Hausdorff distance $H(A, B) . A$ and $B$ are the automatically estimated lung region and the corresponding manual segmentation serving as ground truth.

\begin{tabular}{l|cccc} 
Measures/Methods & 4D-Seg & 3D-Seg & Conv & Comb \\
\hline & Normal lungs/Lungs with small tumors $<13 \mathrm{~cm}^{3}$ \\
$J(A, B)$ & $0.92 \pm 0.03$ & $0.92 \pm 0.03$ & $0.95 \pm 0.02$ & $0.95 \pm 0.02$ \\
$\bar{D}(A, B)[\mathrm{mm}]$ & $1.32 \pm 0.48$ & $1.30 \pm 0.51$ & $0.91 \pm 0.52$ & $0.85 \pm 0.31$ \\
$H(A, B)[\mathrm{mm}]$ & $25.06 \pm 9.53$ & $24.89 \pm 8.67$ & $22.68 \pm 8.98$ & $22.98 \pm 8.86$ \\
\hline & \multicolumn{5}{|c}{ Lungs with large tumors $>13 \mathrm{~cm}^{3}$} \\
$J(A, B)$ & $0.92 \pm 0.03$ & $0.92 \pm 0.03$ & $0.89 \pm 0.04$ & $0.95 \pm 0.02$ \\
$\bar{D}(A, B)[\mathrm{mm}]$ & $1.45 \pm 0.49$ & $1.46 \pm 0.52$ & $2.12 \pm 1.26$ & $0.91 \pm 0.30$ \\
$H(A, B)[\mathrm{mm}]$ & $21.57 \pm 6.02$ & $21.69 \pm 5.93$ & $36.65 \pm 11.03$ & $19.64 \pm 3.63$ \\
\hline
\end{tabular}

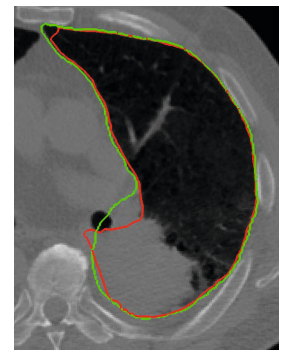

(a) 4D-Seg

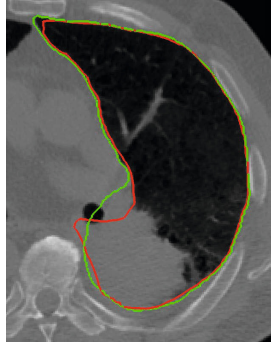

(b) 3D-Seg

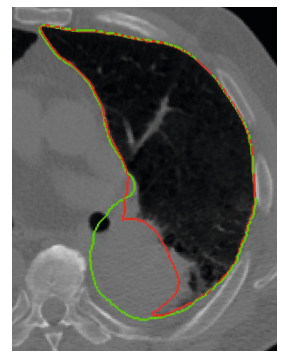

(c) Conv

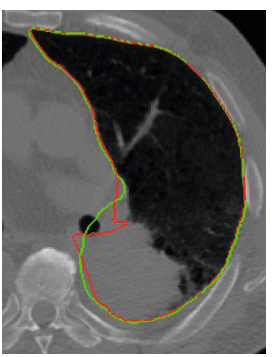

(d) Comb

Fig. 1. Segmentation results for a right lung with a tumor adhering to the chest wall. Results generated with different segmentation methods. Depicted is an axial slice of one breathing phase (EI) of a 4D CT image sequence. Results of the automatic methods are displayed in red, the manually obtained segmentation in green.

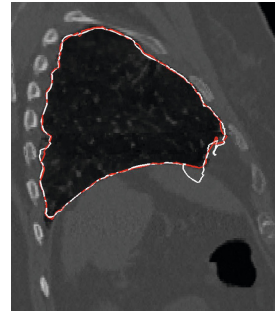

(a) EE

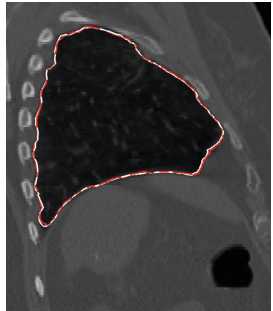

(b) MI

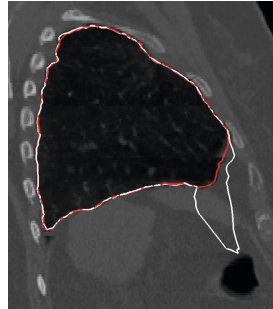

(c) EI

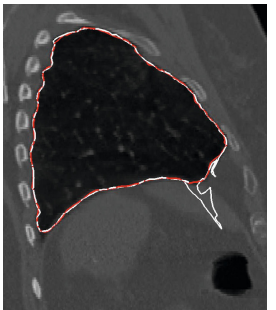

(d) $\mathrm{ME}$

Fig. 2. Left lung segmentation results for 4 different respiratory phases of a 4D CT image sequence obtained by employing 3D-Seg (white contour) and 4D-Seg (red contour) method. Results of 3D-Seg are partially influenced by the gas-filled bowel resulting in temporally inconsistent segmentations. The errors in (c) and (d) are mainly introduced in the 3D-SSM fitting stage. Results of 4D-Seg were computed using all 10 phases of the $4 \mathrm{D}$ data set. 
of EE, MI, EI, and ME. In total, 80 segmentations (normal and small tumors: 60, large tumors: 20) were used as ground truth for the evaluation. The accuracy of the proposed model-based 4D segmentation approach (4D-Seg) was compared to three other approaches: 1) standard intensity-based lung segmentation similar to [3] (Conv), 2) standard 3D-SSMs (see eq. (1)) and ASM fitting with spatially consistent optimal surface finding (3D-Seg), and 3) combined results of 4D and standard segmentation $(\mathrm{Comb}=4 \mathrm{D}-\mathrm{Seg} \cup \mathrm{Conv})$. Three error metrics were utilized for the comparison: Jaccard coefficient, symmetric mean surface distance, and the symmetric Hausdorff distance. The parameters of method 4D-Seg were fixed for all test cases $\left(L=20, \delta=1.0 \mathrm{~mm}, \Delta_{3 D}=\Delta_{4 D}=10, a=0.01\right)$. For the $3 \mathrm{D}-\mathrm{Seg}$ method, we were unable to determine common parameter values suitable for all test cases $\left(L=15-30, \delta=1.0 \mathrm{~mm}, \Delta_{3 D}=10, a=0.01-0.015\right)$.

Table 1 summarizes the quantitative results of our evaluation grouped into two categories (normal and small tumors, large tumors). It can be observed that standard segmentation (Conv) leads to better results for normal lungs and lungs with small tumors than the model-based approaches 4D-Seg and 3D-Seg. As shown in Fig. 1, in the case of lungs with large tumors adhering to nonlung structures, Conv is outperformed by 4D-Seg and 3D-Seg, which give nearly equivalent overall accuracy. The small differences between them reported in Tab. 1 are not statistically significant (paired t-test, $p<0.05$ ). However, despite that, Fig. 2 depicts an exemplary case where only the 3D approach is partially influenced by the gas-filled bowel resulting in temporally inconsistent segmentations. Using 3D-Seg with a single set of parameters for all patients $(L=30, a=0.01)$ leads to two additional cases with temporal inconsistencies.

Due to the globally chosen smoothness constraints and the small training set used for model building, both model-based approaches are prone to cause overand under-segmentation in higly curved areas of the lungs. Therefore, combining the results of $4 \mathrm{D}$ and standard segmentation (Comb) yields the best accuracy for lungs with large tumors, because under-segmentation is considerably reduced.

\section{Conclusion}

In this paper, we present an automatic model-based method to simultaneously segment the lungs in all 3D images of 4D CT data sets of lung cancer patients. This method combines the fitting of a 4D-SSM with a 4D graph-based refinement step, taking into account spatio-temporal consistency. An intrinsic characteristic of the 4D-SSM is that differences within a $4 \mathrm{D}$ sequence are restricted to intrapatient variations and therefore temporal consistency is achieved without explicit temporal regularization. In contrast to [7, our novel 4D model fitting algorithm works without a heuristic pre-initilization of the intra-patient variation parameters. Thus, no assumptions about number and ordering of the breathing phases to be segmented are needed. Furthermore, the 4D-SSM can also be used to segment breathing phases not included in the model's training data set. Experimental results demonstrate the potential of the proposed 4D model-based approach, which performs better than standard intensity-based segmentation in the presence of 
large tumors adhering to non-lung structures. Under-segmentations in highly curved areas of the lungs can be reduced by combining $4 \mathrm{D}$ and conventional segmentation algorithms. While the mean overall accuracy of 3D and 4D modelbased segmentation is nearly identical, improvements in temporal coherence and robustness with respect to the segmentation parameters can be achieved by using the $4 \mathrm{D}$ approach. Summing up, there is no reason to use a 3D approach for lung segmentation in $4 \mathrm{D}$ data sets in the presence of large tumors. This is also supported by the nearly identical running times of both model-based methods ( $\approx 40 \mathrm{~min}$. for a $4 \mathrm{D}$ data set with 14 phases $)$. In future work, the training and test data sets will be significantly enlarged, and we intend to improve the robustness of the least-squares model fitting by adding an outlier detection step.

\section{References}

1. Keall, P.J., Mageras, G., Balter, J.M., et al.: The management of respiratory motion in radiation oncology report of AAPM task group 76. Med. Phys. 33(10), 3874-3900 (2006)

2. Armato, S.G., Sensakovic, W.F.: Automated lung segmentation for thoracic CT: Impact on computer-aided diagnosis. Acad. Radiol. 11(9), 1011-1021 (2004)

3. Hu, S., Hoffman, E., Reinhardt, J.: Automatic lung segmentation for accurate quantitation of volumetric X-ray CT images. IEEE Trans. Med. Imaging 20(6), 490-498 (2001)

4. Sluimer, I., Prokop, M., van Ginneken, B.: Towards automated segmentation of the pathological lung in CT. IEEE Trans. Med. Imaging 24(8), 1025-1038 (2005)

5. van Rikxoort, E., de Hoop, B., Viergever, M., Prokop, M., van Ginneken, B.: Automatic lung segmentation from thoracic computed tomography scans using a hybrid approach with error detection. Med. Phys. 36(7), 2934-2947 (2009)

6. Sun, S., Bauer, C., Beichel, R.: Automated 3-d segmentation of lungs with lung cancer in CT data using a novel robust active shape model approach. IEEE Trans. Med. Imaging 31(2), 449-460 (2012)

7. Perperidis, D., Mohiaddin, R., Edwards, P., Rueckert, D.: Segmentation of cardiac $\mathrm{mr}$ and ct image sequences using model-based registration of a $4 \mathrm{~d}$ statistical model. In: Pluim, J.P.W., Reinhardt, J.M. (eds.) Proc. SPIE Medical Imaging 2007: Image Processing. SPIE, vol. 6512, p. 65121D (2007)

8. Ehrhardt, J., Werner, R., Schmidt-Richberg, A., Handels, H.: Statistical modeling of $4 \mathrm{D}$ respiratory lung motion using diffeomorphic image registration. IEEE Trans. Med. Imaging 30(2), 251-265 (2011)

9. Cootes, T.F., Taylor, C.J., Cooper, D.H., Graham, J.: Active shape models their training and application. CVIU 61, 38-59 (1995)

10. Mori, K., Hasegawa, J., Toriwaki, J., Anno, H., Katada, K.: Recognition of bronchus in three-dimensional X-ray CT images with application to virtualized bronchoscopy system. In: Pluim, J.P.W., Reinhardt, J.M. (eds.) Proc. 13th Int. Conf. Pattern Recognition, vol. 3, pp. 528-532. IEEE Computer Society (1996)

11. Song, Q., Wu, X., Liu, Y., Smith, M., Buatti, J., Sonka, M.: Optimal Graph Search Segmentation Using Arc-Weighted Graph for Simultaneous Surface Detection of Bladder and Prostate. In: Yang, G.-Z., Hawkes, D., Rueckert, D., Noble, A., Taylor, C. (eds.) MICCAI 2009, Part II. LNCS, vol. 5762, pp. 827-835. Springer, Heidelberg (2009) 